\title{
The detection of iron sulfides in Planetary Nebulae ${ }^{\star}$
}

\author{
S. Hony ${ }^{1}$, J. Bouwman ${ }^{2}$, L. P. Keller ${ }^{3}$, and L. B. F. M. Waters ${ }^{1,4}$ \\ 1 Astronomical Institute "Anton Pannekoek”, University of Amsterdam, Kruislaan 403, 1098 SJ Amsterdam, The Netherlands \\ 2 CEA, DSM, DAPNIA, Service d'Astrophysique, CEN Saclay, 91191 Gif-sur-Yvette Cedex, France \\ 3 Mail Code SR, NASA Johnson Space Center, Houston, Texas 77058, USA \\ ${ }^{4}$ Instituut voor Sterrenkunde, Katholieke Universiteit Leuven, Celestijnenlaan 200B, 3001 Heverlee, Belgium
}

Received 9 July 2002 / Accepted 29 August 2002

\begin{abstract}
We present and discuss the detection, through mid IR spectroscopy, of iron sulfides in the carbon rich ejecta of evolved stars. We find the spectroscopic signature of iron sulfides at $23 \mu \mathrm{m}$. We also find weak features at $\sim 34,38$ and $44 \mu \mathrm{m}$. The positions of these features correspond well with the resonances of the iron sulfide troilite. However, the relative strength of the $23 \mu \mathrm{m}$ versus the other bands does not match the laboratory measurements, which suggests the presence of other iron sulfides besides troilite. The same broad feature around $23 \mu \mathrm{m}$ has been found in young stellar objects. This detection may imply a carbon star origin for part of the iron sulfides found in meteorites and interplanetary dust particles.
\end{abstract}

Key words. circumstellar matter - Planetary Nebulae: individual: PN M2-43, PN K3-17 - stars: mass-loss

\section{Introduction}

Infrared (IR) spectroscopy is an important tool to study the composition of the circumstellar envelopes (CSEs) around stars at various stages of evolution. Evolved stars shed their envelopes. The composition of their CSE reflects the physical and chemical conditions in the extended atmosphere of these stars. The CSE of young stellar objects (YSOs) holds information on the molecular cloud composition, the material which formed the stars and possibly their planetary systems. The building blocks of our solar system can also be studied through different methods. Most notably by studying meteorites, comets and interplanetary dust particles (IDPs). These solar system objects contain materials which are believed to be unaltered during the formation of our solar system. Iron sulfides are an important constituent of all these solar-system building blocks (e.g. Kerridge 1976; Lawler et al. 1989; Dai \& Bradley 2001).

We are studying the IR spectra of planetary nebulae $(\mathrm{PNe})$ to measure which materials are produced in the outflows around evolved stars and fed into the interstellar medium (ISM). In this Letter we present and discuss the detection of a broad feature at $23 \mu \mathrm{m}$ and weaker features at 34 , 38 and 44 in two C-rich PNe. A similar broad feature is found in the spectra of YSOs (Keller et al. 2002). Based on the correspondence with the laboratory spectrum of the iron sulfide

Send offprint requests to: S. Hony,

e-mail: hony@astro.uva.nl

* Based on observations obtained with ISO, an ESA project with instruments funded by ESA Member states (especially the PI countries: France, Germany, The Netherlands and the UK) with the participation of ISAS and NASA. troilite we claim iron sulfides to be a constituent of these PNe. The spectroscopic match is not perfect and we present possible explanations for the observed discrepancies.

\section{The observations}

We present the mid IR spectra of two PNe. M2-43 and K3-17 were observed with the Short Wavelength Spectrometer (SWS) (de Graauw et al. 1996) on-board the Infrared Space Observatory (ISO) (Kessler et al. 1996) in observing mode AOT01 (TDT 14900804 and 49900640). The data were processed using SWS interactive analysis product; IA (see de Graauw et al. 1996) using calibration files and procedures equivalent to pipeline version 10.1. Further data processing consisted of bad data removal and rebinning on a fixed resolution wavelength grid. The match between the individual sub-bands is excellent for K3-17. For M2-43 the data of subband $3 \mathrm{~A}(12.5-16.5 \mu \mathrm{m})$ are systematically too low compared to the neighbouring sub-bands. We have shifted sub-band $3 \mathrm{~A}$ by $+7 \mathrm{Jy}$. The detection of the feature we discuss here is not influenced by this shifting.

\section{Description of the spectrum}

We show the SWS spectrum of the prototypical C-rich PN NGC 7027 in Fig. 1a. We show the spectra of M2-43 and K3-17 from 10 to $45 \mu \mathrm{m}$ in Fig. 1b. The mid IR spectra of these nebulae are typified by two broad emission features: one centred on $\sim 23 \mu \mathrm{m}$, and the other at $34 \mu \mathrm{m}$. The spectrum of M2-43 shows some weak structures on top of both these features with local maxima at 15.5, 20, 22.3, 38 and $44 \mu \mathrm{m}$. The structure 

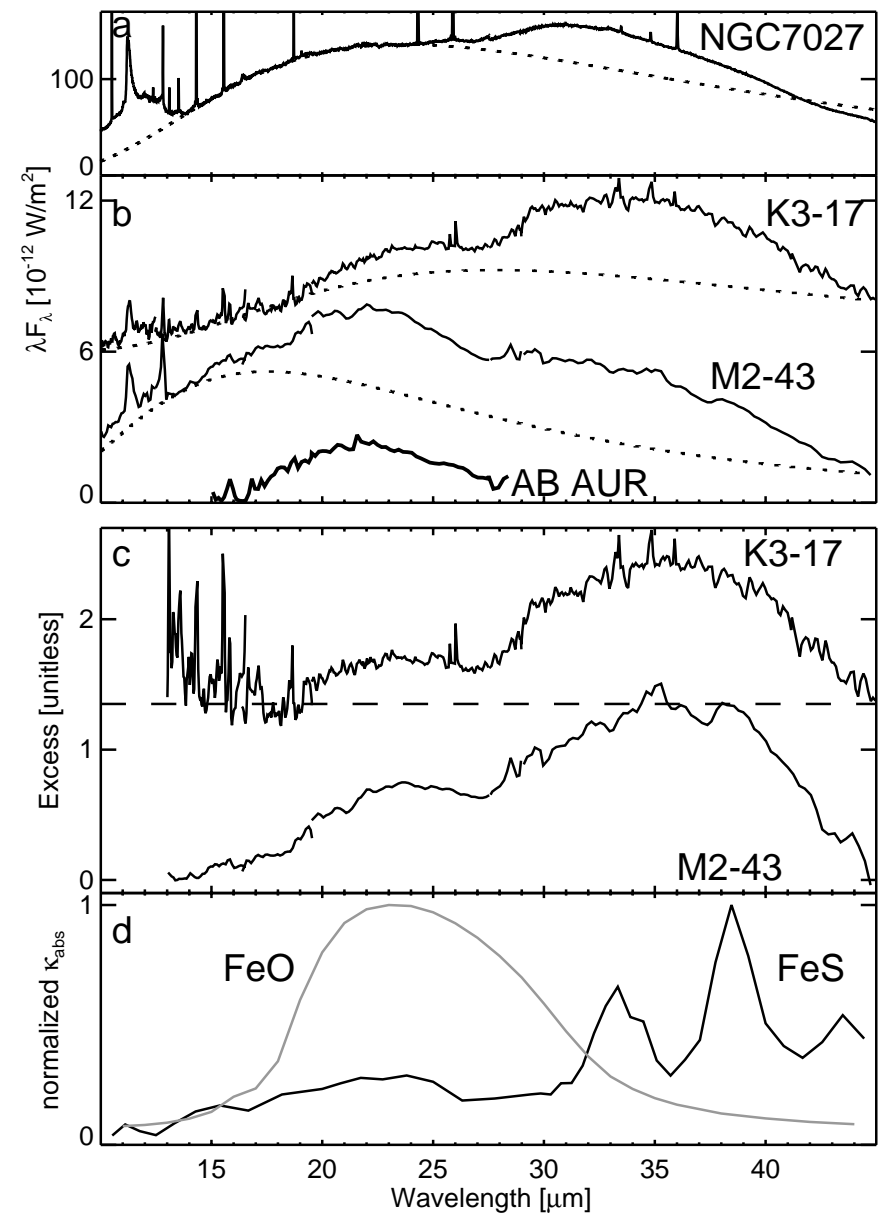

Fig. 1. The SWS spectra of the nebulae exhibiting the $23 \mu \mathrm{m}$ feature are shown in panel b). Panel a shows a typical C-rich PN without the $23 \mu \mathrm{m}$ feature. The model continua are indicated in the panels a) and b) by dotted lines. The spectrum of M2-43 is offset for clarity. We show the feature found in AB Aur for comparison (solid grey line). Panel c) shows the ratio of the excess flux to the continuum flux. The excess of K3-17 is offset for clarity and the zero level is indicated by the dashed line. Panel d) shows the normalised $\kappa_{\text {abs }}$ of FeS (black line) and $\mathrm{FeO}$ (grey line) particles with a $\mathrm{CDE}$ shape distribution.

near $28-29 \mu \mathrm{m}$ is unreliable. We construct a simple model continuum by fitting a modified blackbody $\left(F_{v}(\lambda)=B_{v}(\lambda, T) \times \lambda^{-p}\right.$, where $B_{v}(\lambda, T)$ is the Planck function) to continuum points around $9-10,16$ and $45 \mu \mathrm{m}$. We keep the power $(p)$ fixed at 1.0, a value which in general fits the mid IR spectra of $\mathrm{C}$-rich $\mathrm{PNe}$ well. These continua are shown in Figs. 1a, b and the excess emission above the continuum is shown in Fig. 1c.

A very broad emission feature extending from $25-45 \mu \mathrm{m}$ is abundantly detected in IR spectra of a variety of C-rich evolved objects ranging from intermediate mass loss AGB stars to PNe (Forrest et al. 1981). The feature is attributed to $\mathrm{MgS}$ (e.g. Nuth et al. 1985; Goebel \& Moseley 1985; Hony et al. 2002). In the PNe this feature usually peaks near $34 \mu \mathrm{m}$, see Fig. 1a for a clear example of the feature. The broad feature in M2-43 and K3-17 strongly resembles the feature found in other PNe except for the weak substructures in the 30-45 $\mu \mathrm{m}$ range detected in M2-43.
The feature peaking near $23 \mu \mathrm{m}$ is not found in other PNe. A broad feature like this is however found in the CSE of YSOs where it had previously been ascribed to $\mathrm{FeO}$ (Bouwman et al. 2000). However, we identified this feature with iron sulfides (Keller et al. 2002). We show for comparison the feature of the YSO; AB Aur in Fig. 1b. The feature is obtained after subtracting a radiative transfer model from the SWS spectrum. The feature found in the YSO is very close to the feature found in the PNe, which shows that M2-43 and K3-17 contain the same solid state component as is found around the YSOs and which causes the $23 \mu \mathrm{m}$ feature in both environments.

\section{Comparison with laboratory data}

Begemann et al. (1994) measured the IR reflectance of troilite $(\mathrm{FeS})$. These authors find four strong resonances in the wavelength range we discuss here, a broad feature peaking at $23 \mu \mathrm{m}$ and narrower features at 33, 38 and $43 \mu \mathrm{m}$. We show the emission from $\mathrm{FeS}$ in a continuous distribution of ellipsoids (CDE) shape distribution in Fig. 1c. The feature at $23 \mu \mathrm{m}$ matches well with the emission excess found in M243 and $\mathrm{K} 3-17$, also the signatures of the sharper features at 38 and $43 \mu \mathrm{m}$ match well to the weak features found in M2-43. The presence of the $33 \mu \mathrm{m}$ feature is not obvious from the SWS spectrum. We show in Fig. 2a our attempt to remove the contribution of $\mathrm{MgS}$. We have modelled the emission due to $\mathrm{MgS}$ in a optically thin CDE calculation using the optical constants from Begemann et al. (1994). We vary the strength and temperature of the $\mathrm{MgS}$ feature in order to match most of the $34 \mu \mathrm{m}$ component. We find a temperature of $150 \mathrm{~K}$. This temperature appears very reasonable given the fitted continuum temperature of $165 \mathrm{~K}$. Figure $2 \mathrm{~b}$ shows the residue after removing both the continuum contribution and the emission due to the $\mathrm{MgS}$ component. In the residue a feature near $34 \mu \mathrm{m}$ is also present.

Based on the correspondence in feature positions between the laboratory spectrum and the astrophysical spectra we identify $\mathrm{FeS}$ as the carrier of the $23 \mu \mathrm{m}$ feature. However it is clear from a comparison between Fig. 1 panels $b$ and $c$ that the band strength ratio (BSR) for $\mathrm{FeS}$ and the nebular spectra do not correspond well. In the laboratory spectrum the narrow long wavelength features are much stronger than observed in these nebulae. Below we discuss possible effects, that influence the BSRs.

Firstly, the temperature of the emitting grains influences the observed BSRs. Warmer grains emit stronger towards shorter wavelengths, thereby increasing the strength of the $23 \mu \mathrm{m}$ band relative to the other bands. However, increasing the temperature to $720 \mathrm{~K}$ makes the $23 \mu \mathrm{m}$ band only equally strong in peak strength to the $38 \mu \mathrm{m}$ band. The evaporation temperature of FeS is $720 \mathrm{~K}$. Increasing the temperature much beyond the typical continuum temperature of $200 \mathrm{~K}$, enhances the blue side of the broad $23 \mu \mathrm{m}$ band contrarily to what is observed in the nebular spectra. We conclude that a simple temperature effect cannot explain the differences in the BSRs.

Secondly, the BSRs may be influenced by the particle shape. We compare in Fig. 3a the mass absorption coefficient $\left(\kappa_{\mathrm{abs}}\right)$ of spherical particles and from a CDE-shape distribution. As can be seen the resonances are slightly shifted but the BSRs are hardly affected. 


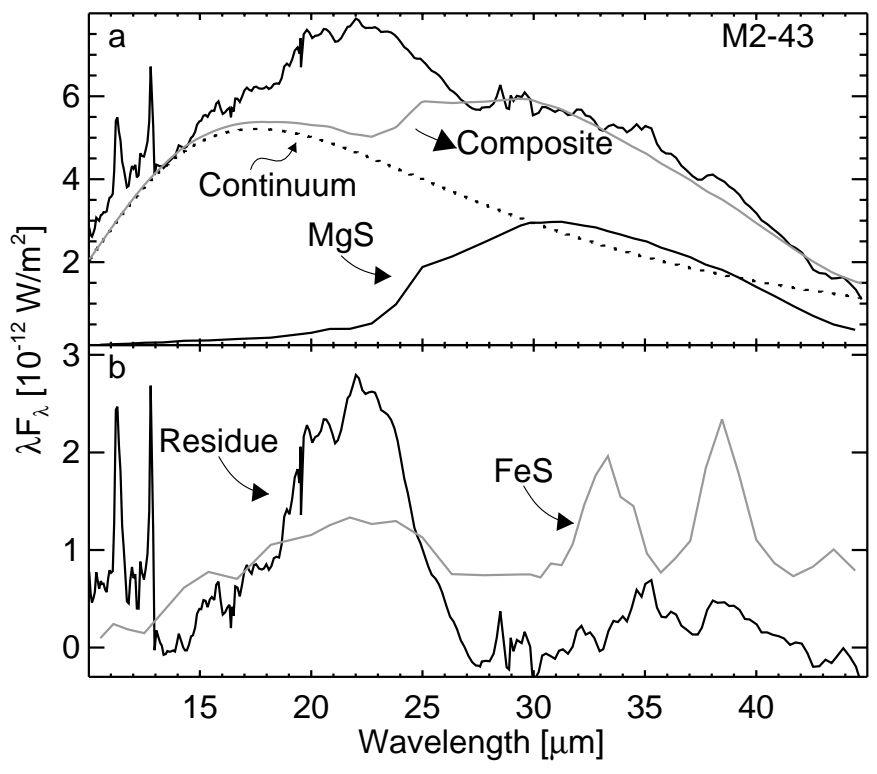

Fig. 2. The mid IR spectrum M2-43. We show in panel a the modelled continuum (dashed), the modelled emission of $\mathrm{MgS}$ at a temperature of $150 \mathrm{~K}$ (black) and the composite modelled spectrum (grey drawn). In panel b) we show the residue after subtracting the composite model from the observed spectrum. In a comparison with FeS (CDE) at $T=$ 150 (grey line) we recognise the major resonances of FeS at 23, 34 and $38 \mu \mathrm{m}$.

Thirdly, the particle might not be homogeneous. We simulated the absorption by a FeS grain embedded in a layer of $\mathrm{MgS}$ in the electrostatic approximation following Bohren \& Huffman (1983, chapter 5). The simulated $\kappa_{\text {abs }}$ from FeS grain with a $\mathrm{MgS}$ coating of 10 and 50 per cent by volume are shown in Fig. 3a. The shortest wavelength resonance is strongly enhanced compared to the longer wavelength bands also the $23 \mu \mathrm{m}$ band shifts towards longer wavelength. This behaviour is due to the strong resonance of $\mathrm{MgS}$ at $26 \mu \mathrm{m}$. The grain with a 50 per cent by volume $\mathrm{MgS}$ coating shows only a single broad resonance at $25 \mu \mathrm{m}$.

Lastly, the BSRs can be affected if the material measured in the laboratory does not have the exact same lattice structure or composition as the material emitting in these nebulae. The material measured by Begemann et al. (1994) is troilite $(\mathrm{FeS})$, however the iron sulfides most commonly found in cometary material and interplanetary dust particles is pyrrhotite $\left(\mathrm{Fe}_{(1-x)} \mathrm{S}\right)$ (Tomeoka \& Buseck 1984). Unfortunately there are no published optical constants for pyrrhotite that cover the whole wavelength range of interest. We show the absorbance of pyrrhotite and iron sulfide inclusion from an IDP to $26 \mu \mathrm{m}$ in Fig. 3b. Both spectra show a broad resonance at $23 \mu \mathrm{m}$. In order to further examine the effects of differences due to composition and lattice structure we have used the published optical constants for pyrite $\left(\mathrm{FeS}_{2}\right)$ (Verble \& Wallis 1969). The emission from pyrite grains is shown by the grey line in Fig. $3 \mathrm{~b}$. The dominant band is at $24 \mu \mathrm{m}$. There are some weaker but sharp resonances at 28.5 and $34 \mu \mathrm{m}$. As can be seen by comparing the $\mathrm{FeS}$ and the $\mathrm{FeS}_{2}$ spectra the resonance around $23 \mu \mathrm{m}$ due to the Fe-S stretch is observed in all these iron sulfides.

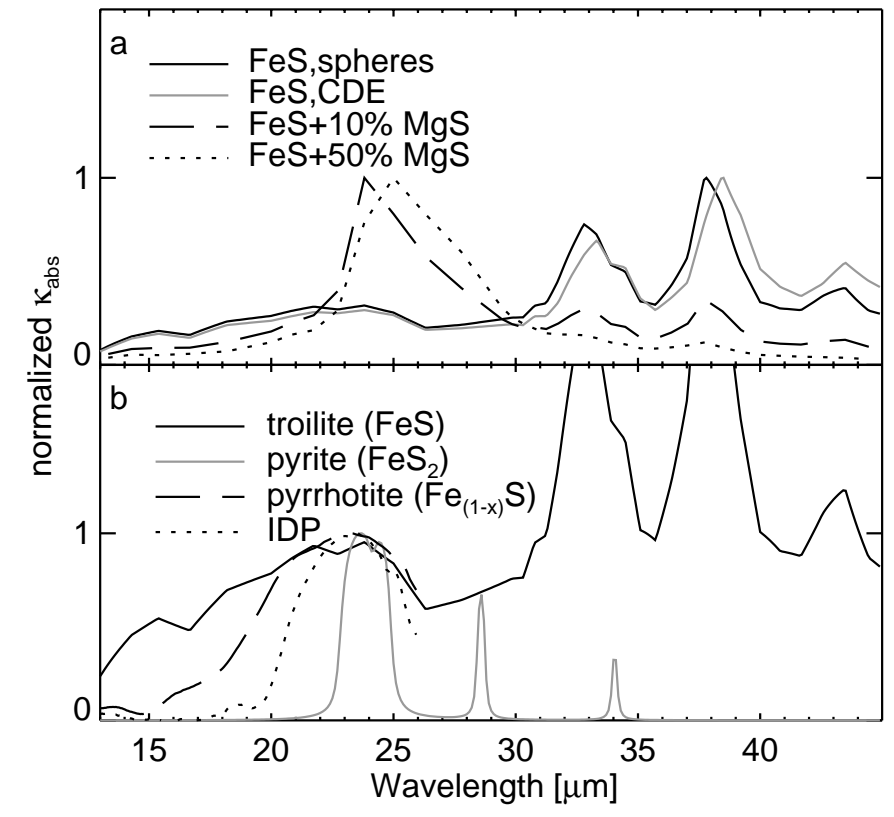

Fig. 3. The effect of particle shape, coating and composition on the optical properties of iron sulfide grains. We show in panel a the normalized $\kappa_{\text {abs }}$ of spherical FeS grains (black line), FeS in a CDE shape distribution (grey line), spherical $\mathrm{FeS}$ grains with a coating of $\mathrm{MgS}$ of 10 percent by volume (dashed line) and 50 per cent by volume (dotted line). In panel b) we show $\kappa_{\mathrm{abs}}$, normalized at $23 \mu \mathrm{m}$ for different types of iron sulfides: $\mathrm{FeS}$ (black line), $\mathrm{FeS}_{2}$ (grey line), $\mathrm{Fe}_{(1-x)} \mathrm{S}$ (dashed line) and the IDP iron sulfide (dotted line). All calculations are done in the Rayleigh limit when the particle radius is much smaller than the wavelength. The temperature of the grains is $200 \mathrm{~K}$.

The longer wavelength bands vary more. One might speculate that the $23 \mu \mathrm{m}$ band is generic to all $\mathrm{Fe}-\mathrm{S}$ bearing materials while the longer wavelength bands are more sensitive to the lattice structure and may be supressed in case the lattice is disorded.

\section{Discussion}

The fact that we find the $23 \mu \mathrm{m}$ feature in the spectra of PNe and YSOs may have implications for the origin of the iron sulfide in our solar system and it puts constraints on the carrier: it must be a plausible component in these chemically very different environments.

Previously it has been suggested that the $23 \mu \mathrm{m}$ feature in YSOs is carried by $\mathrm{FeO}$ (Bouwman et al. 2000). FeO exhibits a single sharp resonance at $20 \mu \mathrm{m}$. This resonance broadens and shifts to $23 \mu \mathrm{m}$ in a CDE shape distribution (Begemann et al. 1995) (e.g. Fig. 1). This indentification relieves the problem with the long wavelength resonances of FeS. Against this identification argues the thermo-dynamical unstablity of $\mathrm{FeO}$. Moreover, $\mathrm{FeO}$ is not expected to form in a $\mathrm{C}$-rich environment. The SWS spectra of M2-43 and K3-17 shown no evidence for O-rich dust like silicates. Instead they show all the typical C-rich dust components: the " $30 " ~ \mu \mathrm{m}$ feature and the emission due to polycyclic aromatic hydrocarbons (Allamandola et al. $1989)$ at $3.3,6.2,7.7$ and $11.3 \mu \mathrm{m}$. Iron sulfides are predicted 
to form in a reducing environment like the outflows of C-rich evolved stars (Lodders \& Fegley 1999).

Similarly, our simulations of the coated FeS cores with $\mathrm{MgS}$ mantles demonstrate a possible way to circumvent the problems with the weak or absent long wavelength resonances, however $\mathrm{MgS}$ is not stable in an the O-rich circumstellar disk of a YSO. Therefore, these calculations are more a proof of concept rather than a direct candidate for carrier identification. A mantle material which has a strong resonance near $23 \mu \mathrm{m}$ enhances the $23 \mu \mathrm{m}$ feature of the iron sulfide core compared to the longer wavelength resonances and brings the BSRs closer to the observed ones. Of course, this material may well be an iron sulfide like pyrrhotite. Such a coated grain is a special case of the mixture of iron sulfide phases discussed above.

An important question concerns the production of FeS in the outflow around carbon stars. Model calculations show that in carbon star atmospheres Fe condenses into metallic iron at high temperatures (Lattimer et al. 1978). At lower temperatures $\mathrm{S}$ condenses into $\mathrm{MgS}$. At even lower temperatures iron sulfides may form through a gas-solid interaction between $\mathrm{H}_{2} \mathrm{~S}$ and the metallic iron. Because the number ratio of $\mathrm{S}$ to $\mathrm{Mg}$ is close to unity, $\mathrm{S}$ is effectively locked up. Based on these calculations iron sulfides are not expected to form abundantly. However these predictions are based on thermochemical equilibrium calculations without taking the dynamics of the outflow into account. One could envision that if the gas cools rapidly during the outflow the condensation of $\mathrm{MgS}$ might not be complete and $\mathrm{S}$ in the form of $\mathrm{H}_{2} \mathrm{~S}$ could still be available to react with the metallic iron. Within this scenario we would conclude that the outflows in M2-43 an K3-17 have cooled more rapidly than those nebulae which do not exhibit the $23 \mu \mathrm{m}$ feature. The fact that we detect the " 30 " $\mu \mathrm{m}$ feature, which is due to $\mathrm{MgS}$ at a strength which is not less than in other C-rich PNe indicates that the $\mathrm{MgS}$ condensation cannot have been very suppressed.

Secondly, the presence of iron sulfide in these nebulae may be due to an unusually high $\mathrm{S} / \mathrm{Mg}$ ratio. However, this is unlikely since neither $\mathrm{S}$ nor $\mathrm{Mg}$ are produced during the evolution of these objects requiring these peculiar abundance patterns to be present throughout the material from which these stars formed.

Thirdly, the difference between "normal" C-rich PNe and those exhibiting the $23 \mu \mathrm{m}$ feature could be the particle size. $1 \mu \mathrm{m}$ sized iron sulfide particles are opaque in the mid IR leaving them undetectable.
Iron sulfides are abundantly found in meteoritic material, in which they are actually the most important $S$ bearing component. Previously iron sulfides have not been detected around evolved stars. This had led to the understanding that all the iron sulfides in our solar system have been produced during the formation of our solar system. The amount of iron sulfides found in meteorites excludes the carbon star atmospheres as their sole production place, but these new finding imply that there may be a presolar source of iron sulfides in our solar system.

Acknowledgements. SH and LBFMW acknowledge financial support from NWO Pionier grant 616-78-333.

\section{References}

Allamandola, L. J., Tielens, A. G. G. M., \& Barker, J. R. 1989, ApJS, 71,733

Begemann, B., Dorschner, J., Henning, T., Mutschke, H., \& Thamm, E. 1994, ApJ, 423, L71

Begemann, B., Henning, T., Mutschke, H., \& Dorschner, J. 1995, Planet. Space Sci., 43, 1257

Bohren, C. F., \& Huffman, D. R. 1983, Absorption and scattering of light by small particles (New York: Wiley, 1983)

Bouwman, J., de Koter, A., van den Ancker, M. E., \& Waters, L. B. F. M. 2000, A\&A, 360, 213

Dai, Z. R., \& Bradley, J. P. 2001, Geochim. Cosmochim. Acta, 65, 3601

de Graauw, T., Haser, L. N., Beintema, D. A., et al. 1996, A\&A, 315, L49

Forrest, W. J., Houck, J. R., \& McCarthy, J. F. 1981, ApJ, 248, 195

Goebel, J. H., \& Moseley, S. H. 1985, ApJ, 290, L35

Hony, S., Waters, L. B. F. M., \& Tielens, A. G. G. M. 2002, A\&A, 390,533

Keller, L. P., Hony, S., Bradley, J. P., et al. 2002, Nature, 417, 148

Kerridge, J. F. 1976, Nature, 259, 189

Kessler, M. F., Steinz, J. A., Anderegg, M. E., et al. 1996, A\&A, 315, L27

Lattimer, J. M., Schramm, D. N., \& Grossman, L. 1978, ApJ, 219, 230

Lawler, M. E., Brownlee, D. E., Temple, S., \& Wheelock, M. M. 1989, Icarus, 80, 225

Lodders, K., \& Fegley, B. 1999, in Asymptotic Giant Branch Stars, IAU Symp. 191, 279

Nuth, J. A., Moseley, S. H., Silverberg, R. F., Goebel, J. H., \& Moore, W. J. 1985, ApJ, 290, L41

Tomeoka, K., \& Buseck, P. R. 1984, Earth and Planetary Sci. Let., 69, 243

Verble, J. L., \& Wallis, R. F. 1969, Phys. Rev., 182, 783 